\title{
RFID NOS CUIDADOS COM A SAÚDE: UMA REVISÃO DE MAPEAMENTO SISTEMÁTICO
}

\author{
Cristiano Manhães de OLIVEIRA ${ }^{1 *}$, Tiago Andrade Muniz TERRA ${ }^{1}$, Edson Terra AZEVEDO FILHO ${ }^{1}$, Mozart \\ Caetano HEYMANN ${ }^{2}$ \& Italo de Oliveira MATIAS ${ }^{3}$
}

1 Universidade Estadual do Norte Fluminense. Campos dos Goytacazes, Rio de Janeiro, Brasil.

2 Universidade Federal Fluminense. Niterói, Rio de Janeiro, Brasil.

3 Universidade Candido Mendes. Campos dos Goytacazes, Rio de Janeiro, Brasil.

*Autor para correspondência.cristianomoliveira@gmail.com

DOI: http://dx.doi.org/10.18571/acbm. 155

\section{RESUMO}

A RFID (Radio Frequency Identification/ Identificação por Rádio Frequência) é uma tecnologia de identificação e captura automática de dados utilizada para monitorar informações ao longo da cadeia de suprimentos, capaz de rastrear ativos e pessoas e mais recentemente na área da saúde. As aplicações na indústria tratamento da saúde, a RFID surgiu com grande potencial não somente pela capacidade de localizar pessoas e equipamentos em tempo real, mas também em proporcionar acesso a dados clínicos para os profissionais de forma precisa, além da melhoria dos processos envolvidos na gestão de ativos, pacientes e equipe envolvida. O presente trabalho busca através de uma revisão de mapeamento sistemático, demonstrar quais são os periódicos, países e palavras-chave mais relevantes, com relação a RFID no tratamento da saúde.

Palavras chave: Identificação por rádio frequência; Mapeamento sistemático.

\begin{abstract}
RFID (Radio Frequency Identification) is a technology for automatic identification and capture of data used to monitor information along the supply chain, able to track assets and people, and more recently in the healthcare. In healthcare industry, RFID has come up with great potential not only for the ability to locate people and equipment in real time, but also in providing access to clinical data to professionals accurately, in addition to improving the processes involved in management of assets, patients and staff involved. The present work searches through a systematic mapping review, to show which journals, countries and keywords are most relevant, regarding RFID in the treatment of health.
\end{abstract}

Keywords: Radio frequency identification; Mapping review.

\section{Introdução}

A identificação por Rádio Frequência (RFID) não é uma tecnologia recente; vem sendo utilizada desde a Segunda Guerra Mundial (USTUNDAG e TANYAS, 2009). Um breve histórico do RFID é mostrado na Quadro 1. 
Quadro 1: Resumo do histórico do desenvolvimento do RFID e sua disseminação.

\begin{tabular}{|l|l|}
\hline \multicolumn{1}{|c|}{ Período } & \multicolumn{1}{c|}{ Eventos } \\
\hline 1940 a 1950 & Primeira utilização da tecnologia, segunda Guerra Mundial; \\
\hline 1950 a 1960 & $\begin{array}{l}\text { Utilização da tecnologia por laboratórios de pesquisa e empresas comerciais devido ao alto } \\
\text { custo e dimensões dos componentes. }\end{array}$ \\
\hline 1960 a 1970 & $\begin{array}{l}\text { Empresas introduziram novos usos de RFID nas aplicações menos complexas e mais } \\
\text { amplamente usadas como, por exemplo para proteger os itens de inventário, tais como } \\
\text { vestuário em lojas de departamento e livros em bibliotecas. }\end{array}$ \\
\hline 1970 a 1980 & $\begin{array}{l}\text { Primeira patente americana para um sistema RFID ativo com memória regravável e patente } \\
\text { para umsistema passivo. Desenvolvimento de sistema para rastreamento de material radioativo } \\
\text { e gado. Início da utilização em HF (High Frequency). }\end{array}$ \\
\hline 1990 a 2000 & $\begin{array}{l}\text { Alguns estados americanos adotaram um sistema de gerenciamento de tráfego baseada no uso } \\
\text { de leitores que poderia detectar etiquetas de protocolo. Utilização da tecnologia na Europa em } \\
\text { pedágios, ferrovias e controle de acesso. Aplicações de pedágios e ferrovias apareceram outros } \\
\text { continentes. Desenvolvimento de circuitos integrados e melhorias com UHF possibilitando } \\
\text { aplicações na cadeia de suprimentos. A tecnologia começa a fazer parte da vida das pess } 0 a s .\end{array}$ \\
\hline Depois de 2000 & $\begin{array}{l}\text { Exigência por parte do Departamento de Defes dos EUA e Walmart, da adoção da tecnologia } \\
\text { pelos seus fornecedores. Redução dos custos da adoção da tecnologia. Utilizaço da tecnologia } \\
\text { no Brasil em algumas empresas, por exemplo HP, Ceitec e Unilever. }\end{array}$ \\
\hline
\end{tabular}

Fonte: (LANDT, 2005; RODRIGUEZ et al., 2012).

RFID é uma tecnologia de identificação e captura automática de dados que é composta de um transponder (etiqueta ou tag), um transceptor (ou leitor) além de um sistema de middleware (MUSA e DABO, 2016).

Baseia-se na utilização de ondas eletrônicas como forma de comunicar os dados de identificação de alguns elementos, como ativos, pessoas e serviços. De acordo com a Fig. 1, as informações sobre a identificação de um objeto (incluindo outras informações possíveis que podem ser monitoradas por sensores tais como temperatura, pressão, etc.) estão registradas nas tags RFID (1), que estão anexadas a itens que se movem ou estão posicionados ao longo da cadeia de suprimentos. As informações nas etiquetas são lidas por um conjunto de sensores (antenas (2) e leitores (3)) por meio de sinais de rádio frequência. Os sensores são normalmente distribuídos em diferentes estágios e em várias posições na cadeia de suprimentos (pontos de controle em centros de distribuição e armazéns, pontos de controle em processos de fabricação e linhas de montagem, etc.) (PEDROSO, ZWICKER e SOUZA, de, 2009).

O middleware pré-processa os dados RFID colhidos pelo transceptor para remover leituras incompletas e leituras múltiplas para o mesmo transponder. Assim, o middleware faz a conexão com hardware e aplicações empresariais RFID (MUSA e DABO, 2016). 


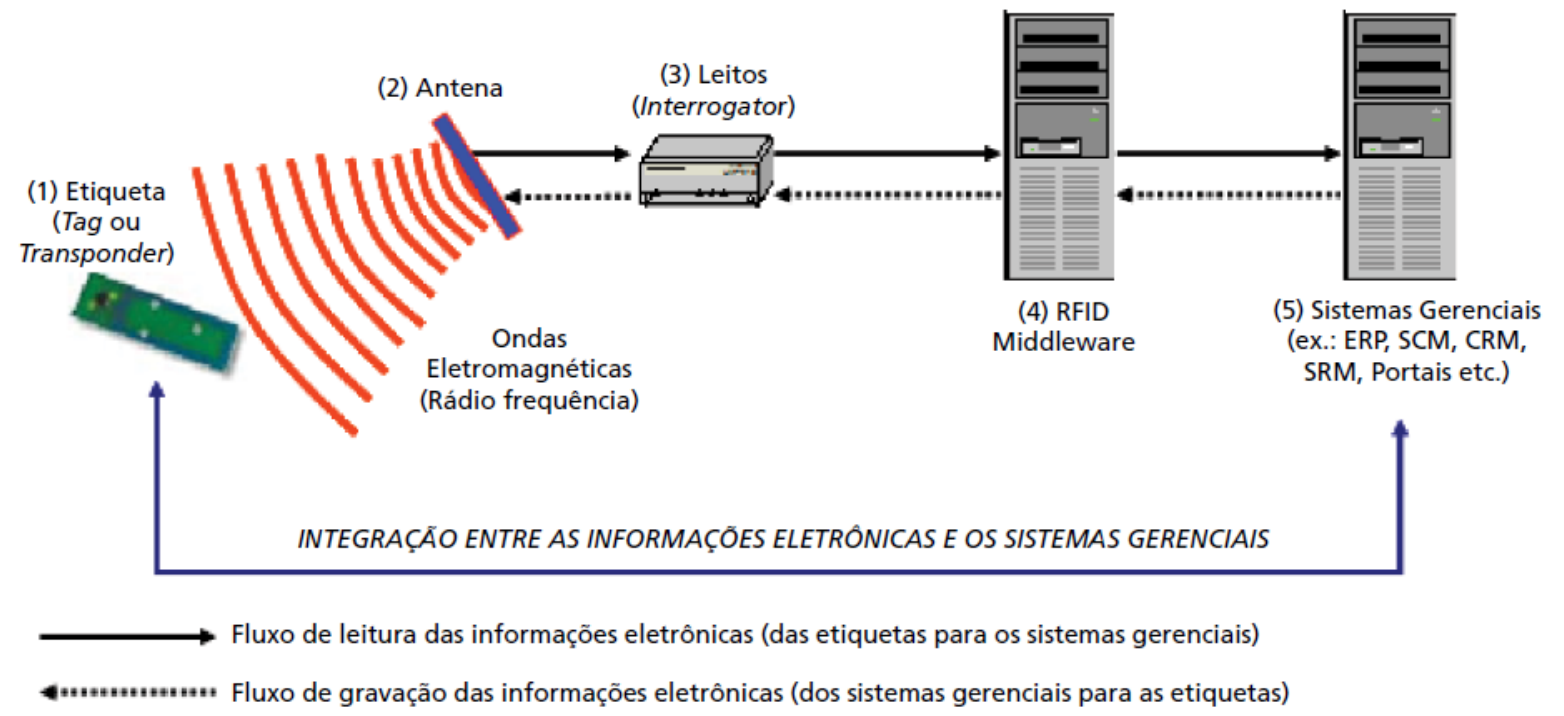

Figura 1: Sistema RFID, Adaptado de: (PEDROSO, ZWICKER e SOUZA, 2009).

RFID tem sido usada para controlar, garantir e monitorar informações ao longo da cadeia de suprimentos (USTUNDAG e TANYAS, 2009), desde o gerenciamento de estoques até o varejo (PEDROSO, ZWICKER e SOUZA, 2009). Essa tecnologia possui aplicações em diversas áreas: bibliotecas (SINGH e MAHAJAN, 2014), aviação (MISHRA e MISHRA, 2010), agricultura (COSTA et al., 2013; RUIZ-GARCIA e LUNADEI, 2011), petróleo (SOARES, P. D. et al., 2017; VALVERDE et al., 2017) e tratamento da saúde (FOSSO WAMBA, ANAND e CARTER, 2013; KUMAR e RAHMAN, 2014; WEN, CHAO-HSIEN e ZANG, 2010).

A indústria de tratamento da saúde é uma das maiores em muitas economias (PAYTON e PARÉ, 2011), além de possuir o potencial de criar 14,3 milhões de postos de trabalho em 2018, todavia, essa indústria tem se deparado com grandes desafios: crescimento dos custos operacionais, alto nível de erros médicos além do envelhecimento da população (FOSSO WAMBA, ANAND e CARTER, 2013).

A tecnologia $R F I D$ além de proporcionar a capacidade para localizar pessoas e equipamentos em tempo real também permite um acesso a dados clínicos para médicos e outros profissionais de forma eficiente e acurada (WEN, CHAO-HSIEN e ZANG, 2010).

$\mathrm{Na}$ saúde a RFID pode proporcionar a melhoria da eficiência em várias formas, como por exemplo através do fluxo físico eficiente (camas, pacientes), acuracidade na informação (registro de tratamentos, histórico dos pacientes) além do gerenciamento de estoques (camas) (BANKS et al., 2007; SARAC, ABSI E DAUZRE-PRS, 2010).

As aplicações da RFID no tratamento da saúde podem ser classificadas nas seguintes áreas (FOSSO WAMBA, ANAND e CARTER, 2013):

- Gestão de ativos: (TZENG, CHEN E PAI, 2008; YANG et al, 2014);

- Gestão de pacientes: (CHEN et al., 2010; CORCHADO, BAJO e ABRAHAM, 2008; TZENG, CHEN e PAI, 2008; XIAO et al., 2006; YANG et al., 2014);

- Gestão da equipe: (CORCHADO, BAJO e ABRAHAM, 2008; TZENG, CHEN e PAI, 2008; XIAO et al., 2006). 


\section{ACTA \\ Biomedica Brasiliensia}

Dessa forma o presente trabalho busca levantar o estado da arte da RFID no tratamento da saúde tendo como base o procedimento adotado por Aria et al., (2017) e Soares et al., (2017).

O trabalho foi organizado como descrito: primeiramente, apresenta-se a metodologia utilizada, que foi uma Revisão de Mapeamento Sistemático (RMS), o que possibilitou a identificação de autores, periódicos e artigos mais relevantes (Seção 2), posteriormente, apresentam-se os resultados (Seção 3) e finalmente as conclusões (Seção 4).

\section{Materiais e Métodos}

Revisões de Mapeamento Sistemático são usadas para estruturar uma área de pesquisa, enquanto que as revisões sistemáticas focam em reunir e sintetizar (PETERSEN, VAKKALANKA e KUZNIARZ, 2015). Existe um número cada vez maior de artigos sendo publicado, o que torna o rastreamento bibliográfico cada vez mais demorado, por outro lado, os RMS estão se tornando um método cada vez mais importante de obter o estado de um campo de pesquisa (KNUTAS et al., 2015).

A extração de dados de produção acadêmica, identificação de padrões bibliométricos, modelagem e visualização das redes de interação entre coautores são tópicos relevantes na área de Bibliometria e Cientometria (MENA-CHALCO e JUNIOR, 2009). A Análise de Redes Sociais (ARS), não é uma teoria formal em sociologia, mas uma estratégia para investigar as estruturas sociais, onde são estudadas as redes de colaboração, além de outras formas de iteração das redes sociais, além disso, a Internet representa uma rede social de uma escala sem precedentes (OTTE e ROUSSEAU, 2002).

Entretanto, hoje em dia, com advento da Internet, encontrar relações entre autores e publicações relevantes, está ficando cada vez mais difícil, devido ao crescente volume de informações (KLINK et al, 2006). As redes de material acadêmico têm grande quantidade de informações. Aproximadamente 114 milhões de documentos científicos (tais como livros, artigos, teses, dissertações e documentos técnicos), somente em inglês, são hoje acessíveis pela web (KHABSA e GILES, 2014).

A utilização da teoria dos grafos para a representação das redes sociais possui algumas vantagens, tais como: representar uma rede social, mensurar precisamente importantes propriedades estruturais, além disso, a representação visual de um grafo permite aos pesquisadores analisarem as interações sociais, descobrir padrões que não foram detectados anteriormente (WASSERMAN e FAUST, 1994). Alguns aplicativos e bibliotecas possuem a capacidade de fornecer uma análise de redes sociais, tais como o Gephi (FAHIMNIA, SARKIS e DAVARZANI, 2015).

Indicadores bibliométricos de ligação e a ARS vêm se consolidando como ferramentas de estudo e de amplo entendimento de fenômenos sociais, como redes de cocitações, estruturas de colaboração científica, redes temáticas de produção científica e outras formas de redes de interação social (OTTE e ROUSSEAU, 2002).

O ranking dos autores é de vital importância para identificar o impacto de um pesquisador num determinado campo científico, para isso existem vários métodos, tais como: número de citações, h-index PageRank e weighted PageRank (DING, 2011a). Dentre esses métodos, o número de citações proposto por Garfield (1955) é o mais comum para mensurar a importância de publicações científicas (MASLOV e REDNER, 2008) e juntamente com journal impact factor, tornou-se um indicador estabelecido para avaliar o impacto de artigos publicados em um periódico (YAN e DING, 2010). Entretanto, o impact factor, e h-index usam contagens de citações e não distinguem citações provenientes de diferentes autores, nem as características das redes de citação (DING, 2011b). 
Bibliometricamente a popularidade, pode ser mensurada como o número de vezes que um autor é citado e prestígio como o número de vezes que um autor é citado em trabalhos altamente citados (DING e CRONIN, 2011), como demonstrado na figura 2.

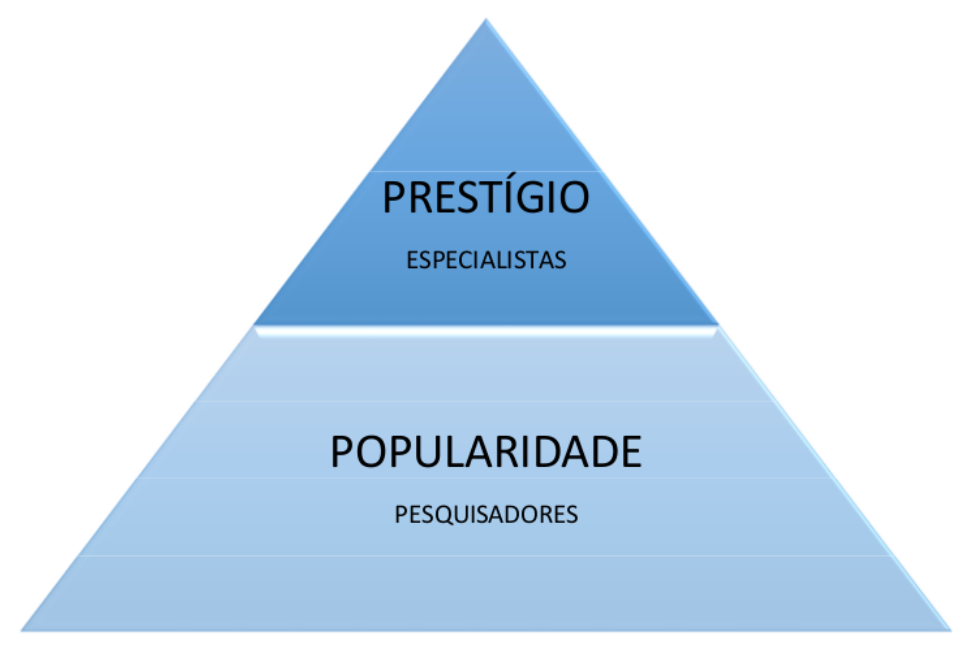

Figura 2: Prestígio e popularidade, Fonte: Adaptado de (DING, 2011b)

Diferentes métodos foram usados no passado para medir a importância de um artigo, o mais comum é o número de citações (DING e CRONIN, 2011). Esse método é o mais comum para mensurar a importância de publicações científicas (MASLOV e REDNER, 2008).

Bibliometricamente a popularidade, pode ser mensurada como o número de vezes que um autor é citado e prestígio como o número de vezes que um autor é citado em trabalhos altamente citados (DING e CRONIN, 2011).

Um artigo altamente citado pode não ser necessariamente um trabalho de prestígio embora em alguns casos possa haver uma forte correlação positiva entre as duas medidas (FAHIMNIA, SARKIS e DAVARZANI, 2015). O uso de citações como métrica de importância possui algumas deficiências como por exemplo a que artigos antigos, porém inovadores, são pouco citados devido à uma comunidade científica menor quando foram publicados, além do fato que publicações sobre descobertas científicas significativas deixam de ser citadas quando seus resultados são incorporados a livros (MASLOV e REDNER, 2008).

O PageRank (BRIN e PAGE, 1998), evita parcialmente tais deficiências, dando maior peso às publicações que são citadas por trabalhos importantes e também identificando um grande número de artigos que contêm resultados inovadores (MASLOV e REDNER, 2008).

Para o desenvolvimento deste trabalho foram adotados métodos similares aos usados por Aria et al. (2017) e Soares et al., (2017). A metodologia é resumida na figura 3: 


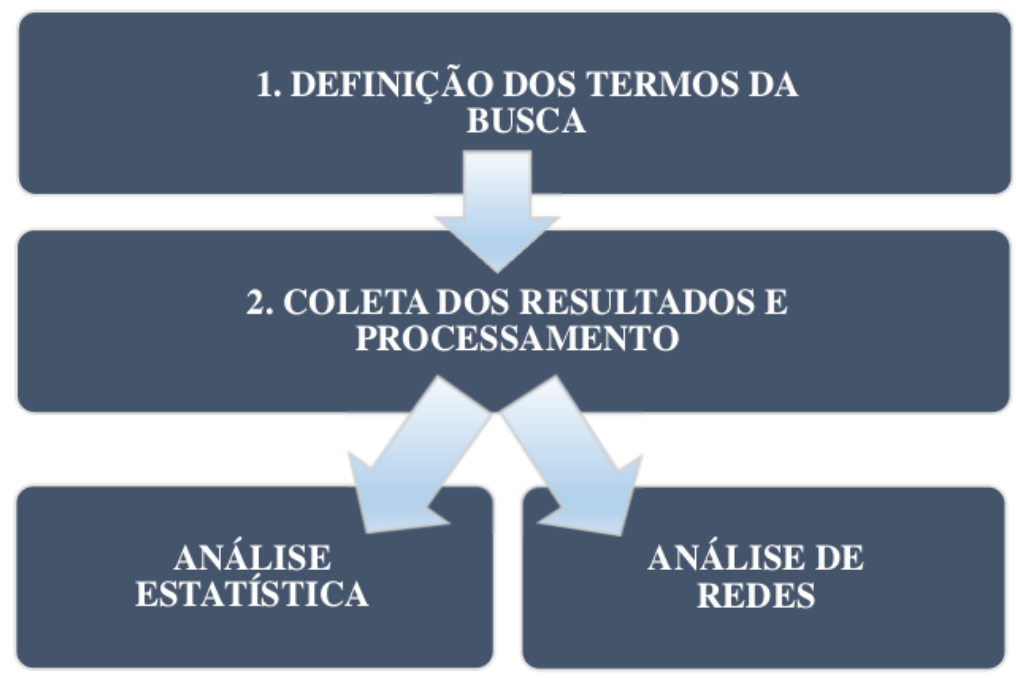

Figura 3: Metodologia utilizada.

Para manipulação dos dados foi utilizada a ferramenta R (R Development Core Team, 2010), juntamente aos pacotes wordcloud (FELLOWS, 2014), rworldmap (SOUTH, 2011) e bibliometrix (ARIA e CUCCURULLO, 2017).

Dessa forma, foi utilizada a base de dados Scopus, por se tratar da mais abrangente (FALAGAS et al., 2007), com a seguinte consulta: "RFID" AND"HEALTHCARE", podendo aparecer no título, resumo ou palavras-chave dos artigos, além do filtro para documentos do tipo "artigos". A pesquisa foi realizada em novembro de 2017 , abrangendo todo o período temporal da base (Tabela 1).

Tabela 1: Coleta dos resultados e processamento.

\begin{tabular}{cc}
\hline Passo/ filtro & $\mathbf{N}^{\circ}$ de artigos \\
\hline Consulta: "RFID" AND "HEALTHCARE” & 855 \\
Filtro: Artigos & 323 \\
Escolha dos relacionados ao tema & \\
\hline
\end{tabular}

\section{Resultados}

Como resultado deste trabalho, obteve-se um resumo dos dados quantitativos da pesquisa, onde primeiramente foi analisada a distribuição cronológica dos artigos para em seguida levantar outras informações relevantes ao tema proposto, tais como: autores, artigos e journals (periódicos), palavras, além de instituições e países que colaboraram entre si.

\subsection{Publicações por Ano}

A figura 4 mostra a distribuição cronológica das publicações: 


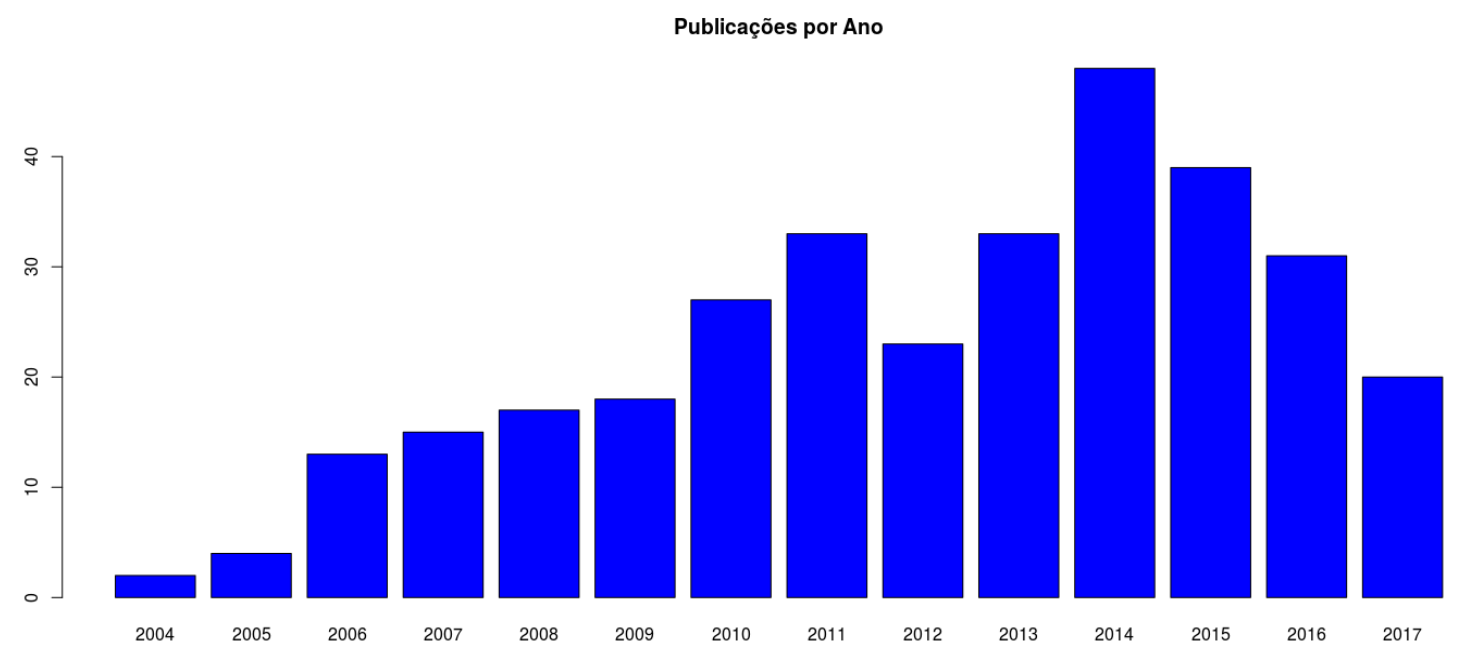

Figura 4: Publicações por ano.

Conforme pode ser observado no gráfico, as pesquisas relacionadas ao tema começaram em 2004 (A PERRIN e SIMPSON, 2004), além disso, o tema obteve um crescimento no número de publicações até 2014, onde posteriormente, ocorreu a redução no número de citações.

O menor número de publicações pode ser justificado pelo fato, que os problemas relacionados a gerenciamento de dados, segurança e privacidade são os temas mais discutidos na atualidade (FOSSO WAMBA, ANAND e CARTER, 2013).

\subsection{Principais periódicos}

O ranking dos periódicos com mais publicações pode ser observado na tabela 2 .

Tabela 2: Ranking dos periódicos que mais publicaram.

\begin{tabular}{lcc}
\hline \multicolumn{1}{c}{ Periódico } & Publicações & CiteScore \\
\hline Journal of Medical Systems & 20 & 2,56 \\
Decision Support Systems & 6 & 4,67 \\
International Journal of Healthcare Technology And Management & 5 & 0,27 \\
International Journal of Distributed Sensor Networks & 4 & 1,16 \\
International Journal of Smart Home & 4 & - \\
International Journal of Bio-Science And Bio-Technology & 4 & - \\
Wireless Personal Communications & 4 & 1,12 \\
International Journal of Rf Technologies: Research And Applications & 4 & 0,84 \\
International Journal of Production Economics & 4 & 4,28 \\
Biomedical Engineering Online & 4 & 2,01 \\
\hline
\end{tabular}


Entre tais periódicos destaca-se o "Journal of Medical Systems", que obteve mais de 3 vezes o número de publicações do segundo colocado, "Decision Support Systems".

\subsection{Principais países}

$\mathrm{Na}$ figura 5 são representados os países mais produtivos.

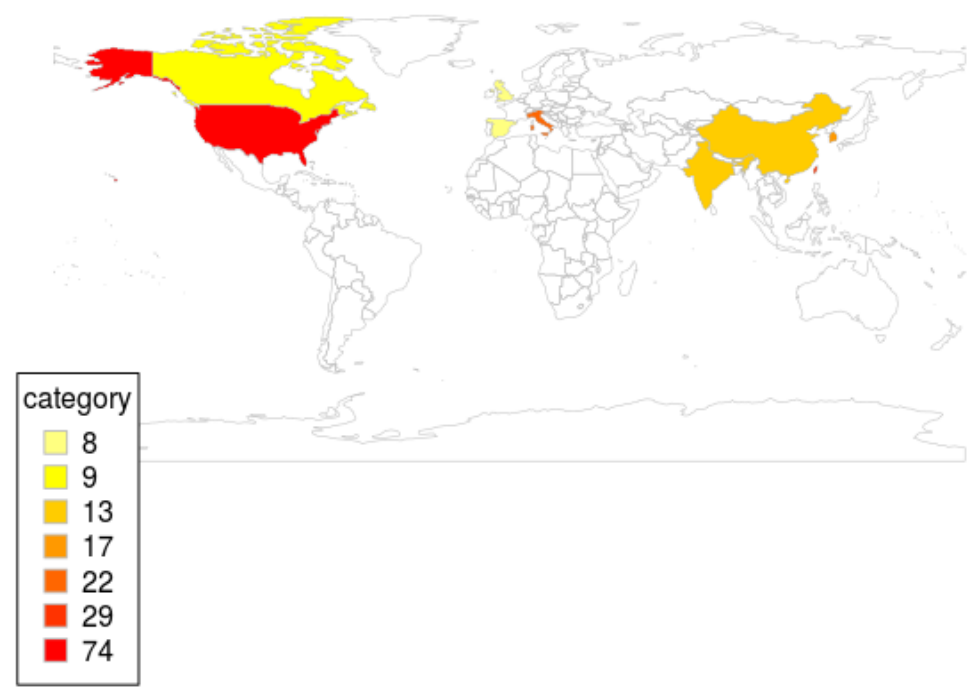

Figura 5: Países com mais publicações.

Na Figura 5, destacam-se os EUA, com 74 publicações, obtendo mais do dobro do segundo colocado, Taiwan com 29 publicações, depois Itália, Coréia e China com 22, 17 e 13 publicações respectivamente.

Outro ponto importante a ser destacado é que os países relacionados ao tema estão concentrados em algumas regiões do globo, como América do Norte, Leste Europeu e Ásia.

A seguir é mostrada a Tabela 3, com o número de citações dos países analisados anteriormente.

Tabela 2: Ranking dos países com relação ao número de citações

\begin{tabular}{cc}
\hline Países & $\mathbf{N}^{\circ}$ de citações \\
\hline EUA & 1465 \\
TAIWAN & 552 \\
ITÁLIA & 387 \\
ESPANHA & 190 \\
HONG KONG & 168 \\
CORÉIA & 161 \\
REINO UNIDO & 132 \\
SUÉCIA & 104 \\
AUSTRÁLIA & 84 \\
CHINA & 76 \\
\hline
\end{tabular}

Conforme pode ser observado na tabela 4, novamente os EUA se destacam obtendo 1.465 citações, ou seja, $44 \%$ de todas as citações, com Taiwan novamente na segunda colocação e Itália em terceiro, por outro lado, a China que ficou na quinta colocação com relação ao número de publicações, obteve a décima colocação quando se trata do número de citações. 


\section{ACTA}

\subsection{Palavras relevantes}

Para uma melhor apresentação dos dados, representa-se na figura 6 uma nuvem de palavras, onde se mostram os termos que possuem um maior número de ocorrências nas palavras-chave dos artigos selecionados. Foram escolhidas aquelas com frequência mínima de 2 e para melhor visualização, termos muitos extensos como "RADIO FREQUENCY IDENTIFICATION" e "RADIO FREQUENCY IDENTIFICATION (RFID)", foram suprimidos e somados ao termo "RFID” por ser tratar de uma abreviação.

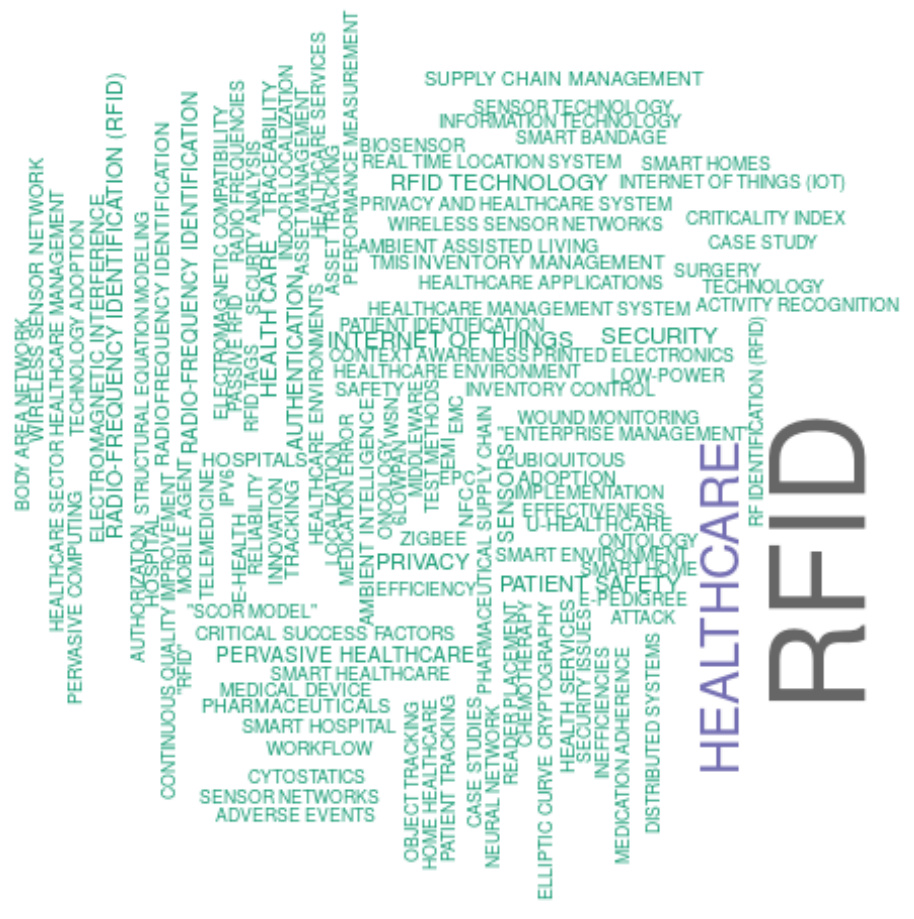

Figura 6: Nuvem de palavras-chave.

Na Tabela 4 são listadas as 10 palavras-chave com maior frequência nos artigos selecionados.

Tabela 4: Ranking das palavras-chave

\begin{tabular}{cc}
\hline Palavras & Frequência \\
\hline RFID & 199 \\
HEALTHCARE & 53 \\
SECURITY & 11 \\
HEALTH CARE & 10 \\
INTERNET OF THINGS & 10 \\
PATIENT SAFETY & 9 \\
PRIVACY & 9 \\
AUTHENTICATION & 7 \\
PERVASIVE HEALTHCARE & 6 \\
SENSORS & 6 \\
\hline
\end{tabular}


Nota-se um destaque para as palavras "RFID" e "HEALTHCARE", que foram os termos utilizados na busca na base SCOPUS. Outro ponto importante a ser ressaltado são as palavras "SECURITY" (segurança) e "PRIVACY" (privacidade), na terceira e sétima colocação que foram apontados por Wamba (2013), como os temas mais discutidos na atualidade com relação a problemas enfrentados na aplicação da RFID.

\subsection{Análise de Redes}

Nesta seção os dados são analisados através da Análise de Redes.

\subsubsection{Colaboração entre países}

Na Figura 7, é representado um grafo de colaboração entre países, onde mais uma vez destacase os EUA.

\section{Colaboração entre Países}

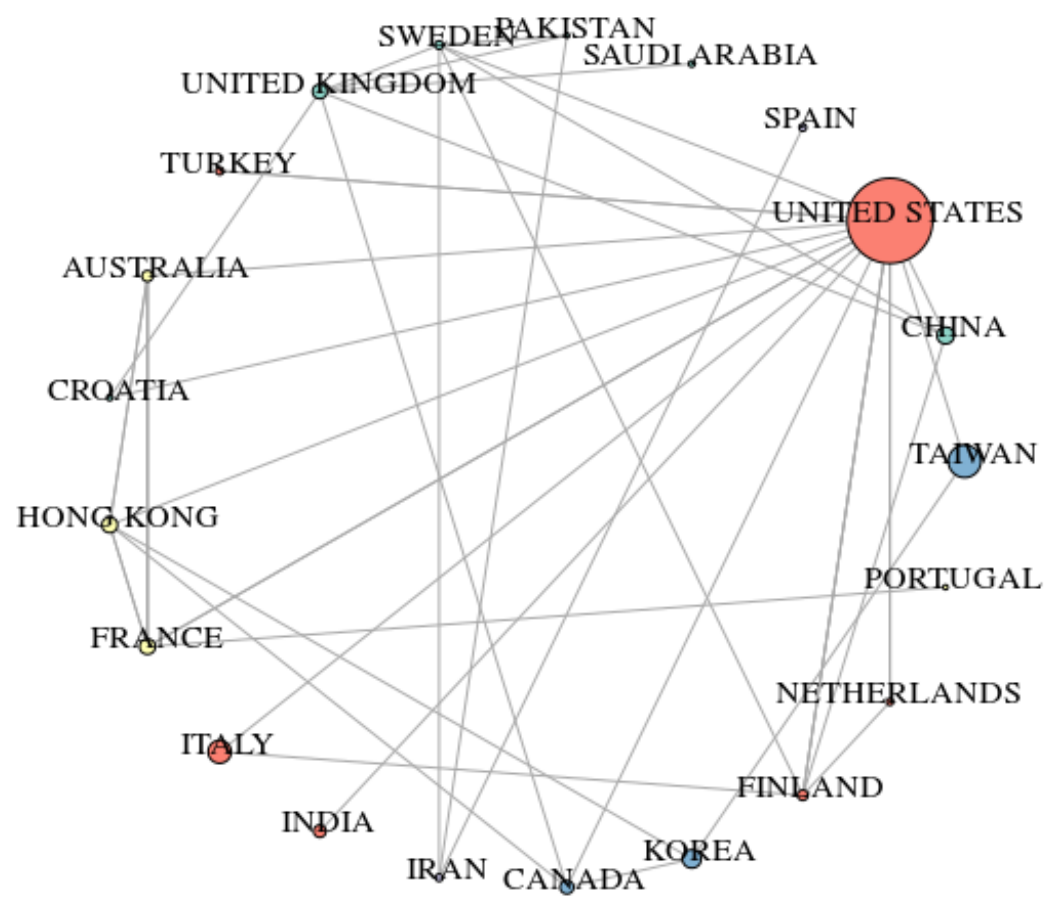

Figura 7: Rede de colaboração entre os países 


\subsubsection{Rede de palavras}

Na Figura 8 é representado um grafo de coocorrência de palavras.

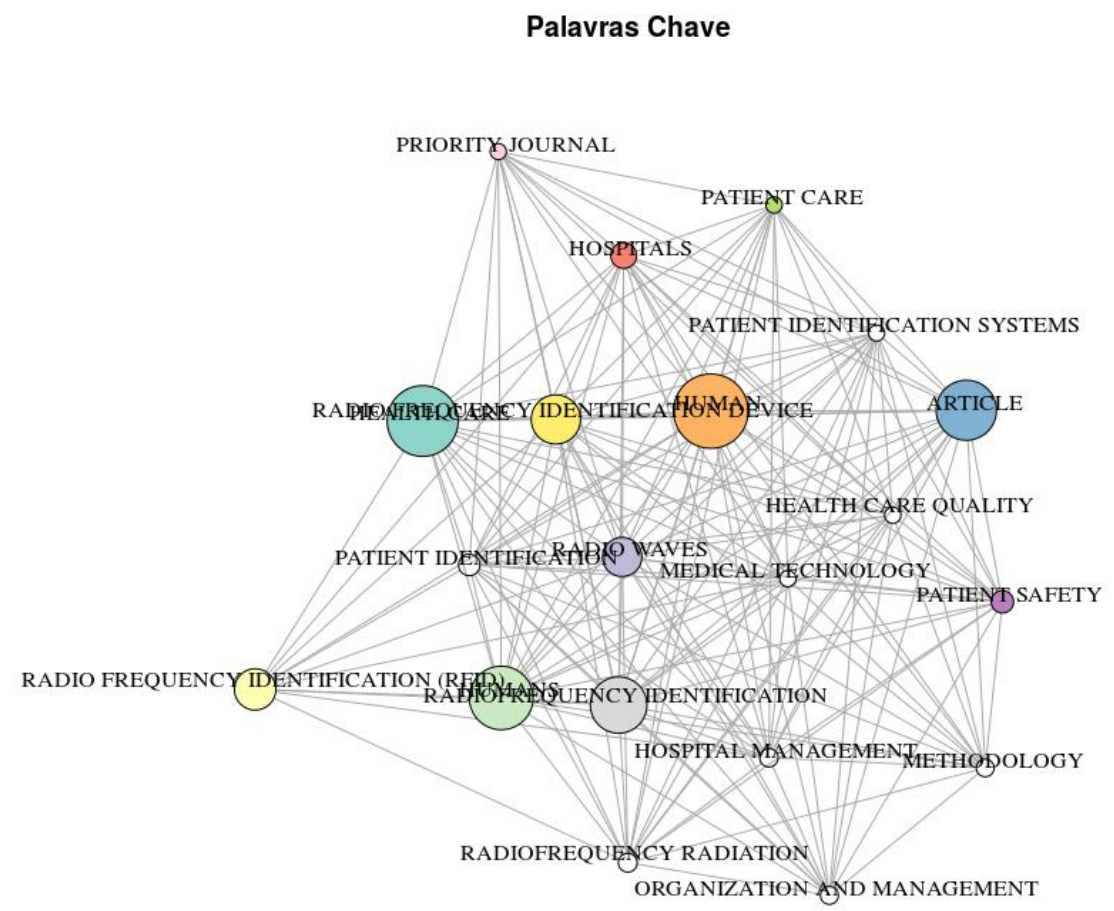

\section{Conclusão}

Figura 8: Coocorrência de palavras-chave.

A RFID não é uma tecnologia recente, com surgimento por volta da Segunda Guerra Mundial, destacou-se em várias áreas com aplicações por toda a cadeia de suprimentos, capaz de rastrear ativos e pessoas e mais recentemente na área da saúde.

As aplicações na indústria tratamento da saúde, a RFID surgiu com grande potencial não somente pela capacidade de localizar pessoas e equipamentos em tempo real, mas também em proporcionar acesso a dados clínicos para os profissionais de forma precisa, além da melhoria dos processos envolvidos na gestão de ativos, pacientes e equipe envolvida.

O presente trabalho demonstrou, o grande interesse da tecnologia no início dos anos 2000 até 2014, onde obteve uma redução das pesquisas com relação ao tema proposto, que pode ser explicado pelos problemas mais discutidos na atualidade, que estão relacionados ao gerenciamento de dados, segurança e privacidade. A tecnologia ainda não é muito utilizada em países como Brasil, em contraste com grande número de trabalhos realizados nos EUA, Ásia e leste Europeu.

Na pesquisa foi utilizado o R para a análise dos dados coletados da base de dados Scopus, que possibilitou agilidade e simplicidade na análise das informações.

Para trabalhos futuros é sugerido a aplicação da metodologia em outras bases como Web of Science e uma análise mais detalhada dos artigos selecionados. 


\section{Referências}

A PERRIN, R.; SIMPSON, N. RFID and bar codes - critical importance in enhancing safe patient care. Journal of healthcare information management: JHIM, v. 18, p. 33-39, 2004.

ARIA, M.; CUCCURULLO, C. bibliometrix: An R-tool for comprehensive science mapping analysis. Journal of Informetrics, v. 11, n. 4, p. 959-975, 2017.

BANKS, J.; HANNY, D.; PACHANO, M. A.; THOMPSON, L. G. RFID Applied. 1. ed. Hoboken, New Jersey: John Wiley \& Sons, 2007.

BRIN, S.; PAGE, L. The anatomy of a large scale hypertextual Web search engine. Computer Networks and ISDN Systems, v. 30, n. 1/7, p. 107-17, 1998.

CHEN, M.; GONZALEZ, S.; LEUNG, V.; ZHANG, Q.; LI, M. A 2G-RFID-based e-healthcare system. IEEE Wireless Communications, v. 17, n. 1, p. 37-43, 2010.

CORCHADO, J. M.; BAJO, J.; ABRAHAM, A. GerAmi: Improving healthcare delivery in geriatric residences. IEEE Intelligent Systems, v. 23, n. 2, p. 19-25, 2008.

COSTA, C.; ANTONUCCI, F.; PAllOtTINO, F.; AGUZZI, J.; SARRIÁ, D.; MENESATTI, P. A Review on Agri-food Supply Chain Traceability by Means of RFID Technology. Food and Bioprocess Technology, v. 6, n. 2, p. 353-366, 2013.

DING, Y. Topic-based PageRank on author cocitation networks. Journal of the American Society for Information Science and Technology, v. 14, n. 4, p. n/a-n/a, 2011a.

Applying weighted PageRank to author citation networks. Journal of the American Society for Information Science and Technology, v. 62, n. 2, p. 236-245, fev. 2011 b.

DING, Y.; CRONIN, B. Popular and/or prestigious? Measures of scholarly esteem. Information Processing and Management, v. 47, n. 1, p. 80-96, 2011.

FAHIMNIA, B.; SARKIS, J.; DAVARZANI, H. Green supply chain management: A review and bibliometric analysis. Intemational Journal of Production Economics, v. 162, n. April, p. 101-114, abr. 2015.

FALAGAS, M. E.; PITSOUNI, E. I.; MALIETZIS, G. A.; PAPPAS, G. Comparison of PubMed, Scopus, Web of Science, and Google Scholar: strengths and weaknesses. The FASEB Journal, v. 22, n. 2, p. 338-342, 2007.

FELLOWS, I. Package “wordcloud”R package version 2.5, 2014. Disponível em: <https //cran.rproject.org/web/packages/wordcloud/index.html>

FOSSO WAMBA, S.; ANAND, A.; CARTER, L. A literature review of RFID-enabled healthcare applications and issues. International Journal of Information Management, v. 33, n. 5, p. 875$891,2013$.

KHABSA, M.; GILES, C. L. The number of scholarly documents on the public web. PLoS ONE, v. 9, n. 5, 2014.

KLINK, S.; REUTHER, P.; WEBER, A.; WALTER, B.; LEY, M. Analysing Social Networks Within 
Bibliographical Data. In: BRESSAN, S.; KÜNG, J.; WAGNER, R. (Eds.). . Database and Expert Systems Applications: 17th International Conference, DEXA 2006, Krak\{ó\}w, Poland, September 4-8, 2006. Proceedings. Berlin, Heidelberg: Springer Berlin Heidelberg, 2006. p. 234 243.

KNUTAS, A.; HAJIKHANI, A.; SALMINEN, J.; IKONEN, J.; PORRAS, J. Cloud-based Bibliometric Analysis Service for Systematic Mapping Studies. Proceedings of the 16th International Conference on Computer Systems and Technologies, p. 184-191, 2015.

KUMAR, A.; RAHMAN, S. RFID-enabled process reengineering of closed-loop supply chains in the healthcare industry of Singapore. Journal of Cleaner Production, v. 85, p. 382-394, 2014.

LANDT, J. The history of RFID. IEEE Potentials, v. 24, n. 4, p. 8-11, 2005.

MASLOV, S.; REDNER, S. Promise and Pitfalls of Extending Google's PageRank Algorithm to Citation Networks. Journal of Neuroscience, v. 28, n. 44, p. 11103-11105, 2008.

MENA-CHALCO, J. P.; JUNIOR, R. M. C. scriptLattes: an open-source knowledge extraction system from the Lattes platform. Journal of the Brazilian Computer Society, v. 15, n. 4, p. 31-39, 2009.

MISHRA, A.; MISHRA, D. Application of rfid in aviation industry: An exploratory review. Promet - Traffic - Traffico, v. 22, n. 5, p. 363-372, 2010.

MUSA, A.; DABO, A. A. A. A Review of RFID in Supply Chain Management: 2000-2015. Global Journal of Flexible Systems Management, v. 17, n. 2, p. 189-228, 2016.

OTTE, E.; ROUSSEAU, R. Social network analysis: a powerful strategy, also for the information sciences. Journal of Information Science, v. 28, n. 6, p. 441-453, 2002.

PAYTON, F. C.; PARÉ, G. Journal of the Association for Information Health Care IT: Process , People, Patients and Interdisciplinary Considerations Health Care IT: Process, People, Patients and. Journal of the Association for Information Systems, v. 12, n. 2, 2011.

PEDROSO, M. C.; ZWICKER, R.; SOUZA, C. A. DE. RFID adoption: framework and survey in large Brazilian companies. Industrial Management \& Data Systems, v. 109, n. 7, p. 877-897, 2009.

PETERSEN, K.; VAKKALANKA, S.; KUZNIARZ, L. Guidelines for conducting systematic mapping studies in software engineering: An update. Information and Software Technology, v. 64, p. $1-18,2015$.

R DEVELOPMENT CORE TEAM. R: A language and environment for statistical computing R Foundation for Statistical Computing Vienna, Austria, 2010. Disponível em: <http://www.rproject.org/>

RODRIGUEZ, L.; HUERTA, M.; ALVIZU, R.; CLOTET, R. Overview of RFID technology in Latin America. Proceedings of the 6th Andean Region International Conference, Andescon 2012, p. 109-112, 2012.

RUIZ-GARCIA, L.; LUNADEI, L. The role of RFID in agriculture: Applications, limitations and challenges. Computers and Electronics in Agriculture, v. 79, n. 1, p. 42-50, 2011.

SARAC, A.; ABSI, N.; DAUZRE-PRS, S. A literature review on the impact of RFID technologies on 
supply chain management. International Journal of Production Economics, v. 128, n. 1, p. 77-95, 2010.

SINGH, N. K.; MAHAJAN, P. RFID and it's use in libraries: A literature review. Intemational Journal of Information Dissemination and Technology, v. 4, n. 2, p. 117, 2014.

SOARES, P. D.; OLIVEIRA, C. M. DE; MORALES, G.; ARICA, J.; MATIAS, I.; FERREIRA, A. S.; CARNEIRO, V. Tracking of Materials and Equipment Used in Planned Maintenance Shutdowns on Offshore Platforms: An Approach using RFIDOTC Brasil. Anais...Offshore Technology Conference, 24 out. 2017Disponível em: <http://www.onepetro.org/doi/10.4043/27951 MS>

SOARES, P. J.; OLIVEIRA, C. M. DE; MORALES, G.; ARICA, J.; MATIAS, I. State of the Art on Arduino and RFIDInternational Joint Conference - ICIEOM-ADINGOR-IISE-AIM-ASEM (IJC 2017). Anais...Valencia, Espanha: 2017

SOUTH, A. rworldmap: A New R package for Mapping Global Data. The R Journal, v. 3, p. 35-43, 2011.

TZENG, S. F.; CHEN, W. H.; PAI, F. Y. Evaluating the business value of RFID: Evidence from five case studies. International Journal of Production Economics, v. 112, n. 2, p. 601-613, 2008.

USTUNDAG, A.; TANYAS, M. The impacts of Radio Frequency Identification (RFID) technology on supply chain costs. Transportation Research Part E: Logistics and Transportation Review, v. 45 , n. 1 , p. 29-38, 2009.

VALVERDE, E.; MURDOCH, E.; WREDEN, C.; GOODWIN, A.; OSEI-KUFFOUR, J. OTC27611-MS Selection and Implementation of RFID Drilling and Completion Technology in Challenging , Deepwater Environments. p. 1-29, 2017.

WASSERMAN, S.; FAUST, K. Social network analysis : methods and applications. Cambridge: Cambridge University Press, 1994.

WEN, Y.; CHAO-HSIEN, C.; ZANG, L. The Use of RFID in Healtcare: Benefits and Barriers. $n$. June, p. 17-19, 2010.

XIAO, Y.; SHEN, X.; SUN, B.; CAI, L. Security and privacy in RFID and applications in telemedicine. IEEE Communications Magazine, v. 44, n. 4, p. 64-72, 2006.

YAN, E.; DING, Y. Weighted citation: An indicator of an article's prestige. Journal of the American Society for Information Science and Technology, 2010.

YANG, G.; XIE, L.; MÄNTYSALO, M.; ZHOU, X.; PANG, Z.; XU, L. DA; KAO-WALTER, S.; CHEN, Q.; ZHENG, L. R. A Health-IoT platform based on the integration of intelligent packaging, unobtrusive bio-sensor, and intelligent medicine box. IEEE Transactions on Industrial Informatics, v. 10, n. 4, p. 2180-2191, 2014. 